\title{
Total Ionization Radiation Sensor Performance Improvement by Using Si-rich MONOS Device
}

\author{
Hsieh Wen-Ching ${ }^{1^{*}}$, Hao-Tien Daniel Lee ${ }^{2}$, Fuh-Cheng Jon ${ }^{3}$, Shich-Chuan Wu ${ }^{4}$ Dawei Heh ${ }^{5}$, Tzu-I Tsai ${ }^{6}$ \\ ${ }^{1}$ Minghsin Universuty of Science and Technology, Xinxing Rd, 1, Xinfeng 30401, Taiwan \\ ${ }^{2}$ Treasure Giant Technology Inc. 3F-1, 42, Lyushuei Rd. Hsinchu City, 30068 Taiwan \\ ${ }^{3}$ Southern Taiwan University of Science and Technology, 1, Nan-Tai Street, Yungkang District, Tainan 710, Taiwan \\ ${ }^{4}$ National Nano Device Laboratories, Hsinchu City, 30068 Taiwan \\ ${ }^{5}$ Taiwan Semiconductor Manufacturing Company Limited, Hsinchu City, 30068 Taiwan \\ ${ }^{6}$ United Microelectronics Corporation, Hsinchu City, 30068 Taiwan
}

\begin{abstract}
The Si-rich metal -oxide-nitride-oxide-silicon (hereafter Si-MONOS) can be candidates for non-volatile total ionization dose (TID) radiation sensors. In the case of Si-MONOS gamma radiation sensors, the gamma radiation induces a significant decrease of threshold voltage $\mathrm{V}_{\mathrm{T}}$. The change of $\mathrm{V}_{\mathrm{T}}$ for Si-MONOS after gamma irradiation has a strong correlation to the TID of gamma ray exposure as well. The Si-MONOS capacitor device in this study has demonstrated the better feasibility for non-volatile TID radiation sensing in the future.
\end{abstract}

\section{Introduction}

Total ionizing dose (TID) radiation effect is the major application concern for the operation of electronic devices in advanced X-ray lithography semiconductor manufacture process and outer space application, as well as other harsh environments such as accelerators, where high and low energy particles exist. When a MOS structure is irradiated by gamma rays, the positive charges build-up at $\mathrm{Si}-\mathrm{SiO} 2$ interface and the interface state occurred [1]. The irradiation effects of a MetalNitride-Oxide- Silicon (MNOS) device with stacked insulation layers composed silicon nitride and silicon dioxide has been reported [2] [5]. However the detailed mechanism of the charged trappings has not been understood yet. In this study, the radiation-induced trapped charges in the insulation layer of Si rich metal oxide-nitride-oxide-silicon (hereafter Si-MONOS) and metal-oxide-nitride-oxide-silicon (hereafter MONOS) capacitor structures are investigated. The gamma ray radiation induces significant decrease of threshold voltage $\mathrm{V}_{\mathrm{T}}$ for both Si-MONOS and MONOS devices. It is considered that this $\mathrm{V}_{\mathrm{T}}$ change mostly owing to significant increase of radiation induced positive trapped charges in the $\mathrm{Si}$ rich oxide-nitride-oxide (hereafter $\mathrm{Si}$ ONO) and oxide-nitride-oxide (hereafter ONO) gate dielectric after gamma ray irradiation, respectively. In this paper, the change of $\mathrm{V}_{\mathrm{T}}$ for both Si-MONOS and MONOS devices after Gamma ray expose were compared; the change of $\mathrm{V}_{\mathrm{T}}$ for Si-MONOS is more significant than that of MONOS. This Si-MONOS capacitor device with ONO gate dielectric in this study

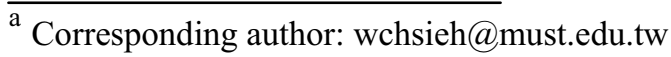

has demonstrated the better potential for the application of non-volatile gamma ray TID dosimeter.

\section{Proposed Mechanism}

In cases of Si-MONOS and MONOS capacitor devices, gamma ray radiation and fixed gate voltage was applied on these Si-MONOS and MONOS capacitor devices simultaneously for gamma ray data writing. When these Si-MONOS and MONOS capacitor structures are irradiated by gamma rays, the electron-hole pairs are generated throughout the gate insulation layers. These free carriers are swept by electric field, and some of them are captured by the charge trap centers, especially at the oxide-nitride $(\mathrm{O}-\mathrm{N})$ interface and silicon- oxide $(\mathrm{S}-\mathrm{O})$ interface. The amount of captured charge depends on the local density and the capture cross section of the trap centers. When a negative gate voltage $\mathrm{VG}$ is applied on these Si-MONOS and MONOS capacitor devices, the holes generated by radiation in the insulation layers are swept toward metal gate side by electric field, and some of the holes are captured by the charge trap centers at the $\mathrm{O}-\mathrm{N}$ interface; however electrons generated by radiation in the insulation layers are swept toward silicon base side by electric field, and some of electrons are captured by the charge trap centers at the O-N interface. As shown in experiment data, the gamma ray radiation induces significant decrease of $\mathrm{V}_{\mathrm{T}}$ for both Si-MONOS and MONOS capacitor devices. It is considered that the amount of trapped holes in gate insulator due to irradiation is greater than the amount of trapped electron. It is suggested that the amount of holes trapped at O-N interface is higher than the amount of electrons trapped at 
$\mathrm{O}-\mathrm{N}$ interface .The trapped charges change the electrical characteristic of these Si-MONOS and MONOS capacitor devices in terms of gate diode capacitor, gate $\mathrm{V}_{\mathrm{T}}$, and drain current. Therefore, the change of $\mathrm{V}_{\mathrm{T}}$ in this case can be correlated to the amount of trapped charges and the total ionization dose (TID) of gamma radiation as well. These trapped charges due to irradiation are accumulated in gate dielectric layer and can't be destroyed by data reading process. In addition, data in these Si-MONOS and SONOS capacitor devices can be erased to original null state by charges injection mechanism. In this paper, the changes of $\mathrm{V}_{\mathrm{T}}$ for Si-MONOS and MONOS after gamma ray expose are compared. As shown in experiment data, the change of $\mathrm{V}_{\mathrm{T}}$ for $\mathrm{Si}-\mathrm{MONOS}$ is more significant than that for MONOS. It is suggested that the amount of positive trapped charges in the SiONO gate dielectric layer is much greater than that in the ONO gate dielectric layer; so the decrease of $\mathrm{V}_{\mathrm{T}}$ for $\mathrm{Si}$ MONOS is more significant than that for MONOS. This Si-MONOS capacitor device is potentially more suitable for the application of gamma ray dosimeter.

\section{Experimental}

N-type MONOS \& Si-MONOS were used and compared in this paper. N-type MONOS capacitor structures were fabricated on p-type resistivity $15-25 \mathrm{ohm}-\mathrm{cm} \mathrm{Si}<100>$ substrate. We use thermal $\mathrm{SiO} 2$ for tunnelling oxide, CVD Si3N4 or Si-rich CVD Si3N4 for trapping layer, CVD TEOS $\mathrm{SiO} 2$ for blocking oxide of ONO gate dielectric. In order to manipulate the $\mathrm{Si}$ composition of the nitride layer, the $\mathrm{SiH} 2 \mathrm{Cl} 2-\mathrm{NH} 3$ gas flow-rate ratio was set differently for standard nitride and uniform Sirich nitride, respectively. The $\mathrm{SiO} 2-\mathrm{Si} 3 \mathrm{~N} 4-\mathrm{SiO} 2$ (hereafter ONO) gate stack consists of a $100 \AA \sim 200 \AA$ thick silicon nitride trapping layer and $50 \AA \sim 150 \AA$ bottom and top silicon oxides. To write radiation data on these MONOS \& Si-MONOS capacity devices, gamma radiation was exposed on these MONOS \& Si-MONOS capacity devices. For the data read, $\mathrm{V}_{\mathrm{T}}$ was measured at room temperature by using a HP4156A parameter analyzer. The experimental results of gate capacitance applied with various gate voltages $(\mathrm{C}-\mathrm{V})$ were obtained by using a computer-controlled HP4284 parameter analyzer, and the $\mathrm{C}-\mathrm{V}$ curves were measured by sweeping $\mathrm{VG}$ together with zero source-and-drain bias condition at room temperature. Figure 2 shows the charge generation and trapping states of the gate dielectric for the $\mathrm{Si}$ MONOS device after ionization radiation exposure.

\section{Results and Discussion}

\subsection{Threshold voltage shift after ionization radiation exposure}

As illustrated in figure 3 it can be seen that the capacity to voltage curves ( $\mathrm{C}-\mathrm{V}$ curve) of MONOS shifted to the left after $10 \mathrm{Mrad}$ of gamma irradiation. This implies that gamma radiation induces a decrease of $\mathrm{V}_{\mathrm{T}}$ for MONOS. The amount of decrease of $\mathrm{V}_{\mathrm{T}}$ is about 2.0 volts. It is considered that the change is due to increase of net positive trapped charges in the ONO gate dielectric layer after gamma irradiation.

From figure 4, it also can be seen that $\mathrm{C}-\mathrm{V}$ curve of Si-MONOS shifted far to the left after 10 Mrad of gamma irradiation. The amount of decrease of $\mathrm{V}_{\mathrm{T}}$ is up to about 2.5 volts. As shown in the experiment data, the change of $\mathrm{V}_{\mathrm{T}}$ for Si-MONOS is more than that for MONOS after 10Mrad TID of gamma irradiation. The dependence of $\mathrm{V}_{\mathrm{T}}$ shift on TID for Si-MONOS is more than that for SONOS after gamma irradiation. It can be explained that the amount of net positive trapped charges due to gamma irradiation in the $\mathrm{Si}-\mathrm{ONO}$ gate dielectric is greater than that in the ONO gate dielectric after gamma irradiation. These radiation-induced shifts in the irradiated device are a combination of two effects; the first effect is a result from the loss of stored charge in the ONO trapping layer and the second effect is due to a build-up of positive charge resulted from asymmetric trapping of electrons and holes in the ONO trapping layer. These two combined effects cause a large $\mathrm{V}_{\mathrm{T}}$ shift in the Si-MONOS but create a smaller $\mathrm{V}_{\mathrm{T}}$ shift in the MONOS. [5],[6]

\subsection{Gate leakage current after $10 \mathrm{Mrad}$ gamma ray exposure}

As illustrated in figure 5 and figure 6 , both can show that the gate oxide leakage current of Si-MONOS and MONOS device did not increase significant after $10 \mathrm{Mrad}$ gamma ray exposure. The gate oxide leakage current of Si-MONOS increase more significant than MONOS after 10Mrad gamma ray exposure.

\section{3. $\mathrm{V}_{\mathrm{T}}$ decay vs. TID}

In both figure 7 and figure 8 , the decrease of $V_{T}$ for MONOS and Si-MONOS are plotted against the total Gamma ray radiation dose TID received. The decay of $\mathrm{V}_{\mathrm{T}}$ for MONOS and Si-MONOS device increases as a function of Gamma TID is indicated in Figure 7 and figure 8 . The effect of gamma TID on the $\mathrm{V}_{\mathrm{T}}$ change on the Si-MONOS device is more significant than that of the MONOS device. The decrease of $\mathrm{V}_{\mathrm{T}}$ in this case can be correlated to the increase of positive trapped charges in the insulator and the increase of exposure TID for Gamma radiation as well. It also shows that decay of $\mathrm{V}_{\mathrm{T}}$ increases more sharply after Gamma irradiation levels up to $100 \mathrm{krad}$ TID. This result is agreed with the previous studies $[5,6]$.

\section{4. $V_{\mathrm{T}}$ stability vs. retention time}

As illustrated in Figure 9 and Figure 10, it also can be seen that the $\mathrm{V}_{\mathrm{T}}$ decay vs time of Si-MONOS after $10 \mathrm{Mrad}$ gamma irradiation are better than that of MONOS. It shows that retention charge loss in SiMONOS device after 10Mrad gamma irradiation is strongly affected by $\mathrm{Si}$ composition ratio and decreases as nitride trapping layer becomes $\mathrm{Si}$ rich. This capacity device with $\mathrm{Si}$ rich ONO gate dielectric in this study has 
demonstrated the better feasibility of using $\mathrm{Si}$ rich MONOS capacity device for gamma ray TID dosimeter.

\section{Conclusions}

As shown in experiment data, the change of $\mathrm{V}_{\mathrm{T}}$ for $\mathrm{Si}$ MONOS is more significant than that of MONOS. The dependencies of $\mathrm{V}_{\mathrm{T}}$ shift on TID in Si-ONO gate dielectric is more than the one in ONO gate dielectric. It can be explained that the amount of positive trapped charges in the Si-ONO gate dielectric layer is greater than the one in the ONO gate dielectric layer; so the decrease of $\mathrm{V}_{\mathrm{T}}$ for Si-MONOS is more than the one for MONOS. It is suggested that the amount of hole trapped at O-N interface in $\mathrm{Si}$ rich $\mathrm{ONO}$ gate dielectric layer is more significant than the one in the ONO gate dielectric layer. This capacity device with $\mathrm{Si}$ rich ONO gate dielectric in this study has demonstrated the better feasibility of using Si rich MONOS capacity device for the application of non-volatile gamma ray TID dosimeter.

\section{Acknowledgments}

The authors would also like to thank National Nano Device Laboratories (NDL), National Tsing Hua University (NTHU), and National Chiao Tung University (NCTU) to provide the instruments for wafer fabrication and testing. This paper was funded in part by the National Science Council (NSC) sponsor.

\section{References}

1. Watanabe, K.; Kato, M.; Okabe, T.; Nagata, M.: IEEE Trans. Nucl. Sci. NS-33, 1216-1222 (1986)

2. Takahashi, Y.; Ohnishi, K.; Fujimaki, T.; Yoshikawa, M.: IEEE Trans. Nucl. Sci. 461578-1585 (1999)

3. Gasperin, A.; Ghidini, G.; Cester, A.; Paccagnella, A. Oxide-nitride-oxide capacitor reliability under heavy-ion irradiation. IEEE Trans. Nucl. Sci. , 54, 1898-1905 (2007)

4. B.; Dockerty, R.; Shaneyfelt, M.; Habermehl, S.; Murray, J. Total dose radiation response of NROMstyle SOI non-volatile memory elements. IEEE Trans. Nucl. Sci. 55, 3202-3205 (2008)

5. Qiao, F.Y.; Yu, X.; Pan, L.Y.: In Proceedings of the 19th IEEE International Symposium on the Physical and Failure Analysis of Integrated Circuits (IPFA), Singapore,( 2-6 July 2012)

6. Hsieh, W.-C.; Lee, H.-T.D.; Jong, F.-C.: Sensors 14 14553-14566 (2014)

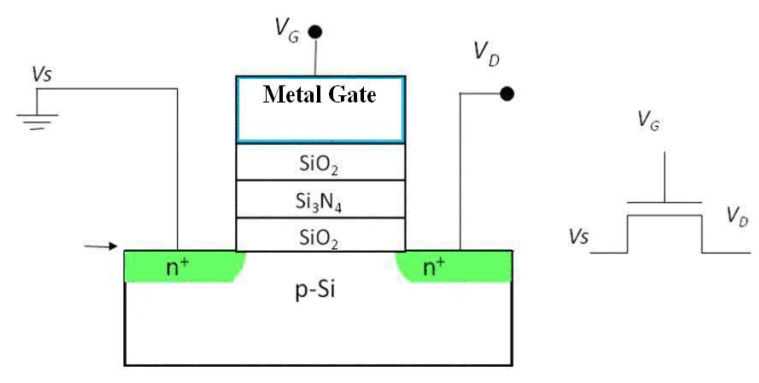

Figure 1. Cross-section view of MONOS devices.

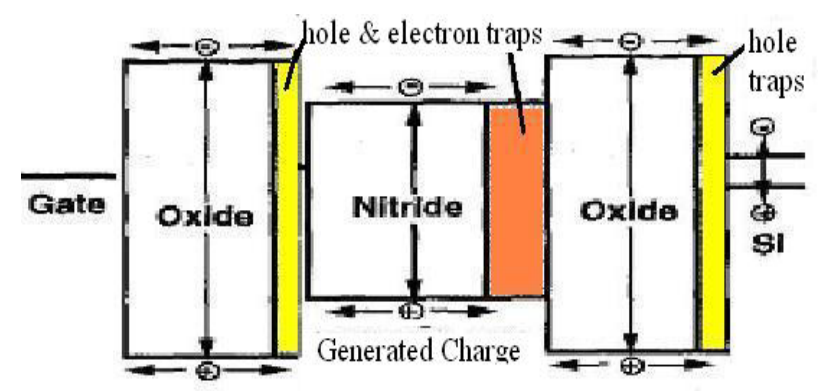

Figure 2. Charges generation and trapping in the MONOS device after Gamma exposure.

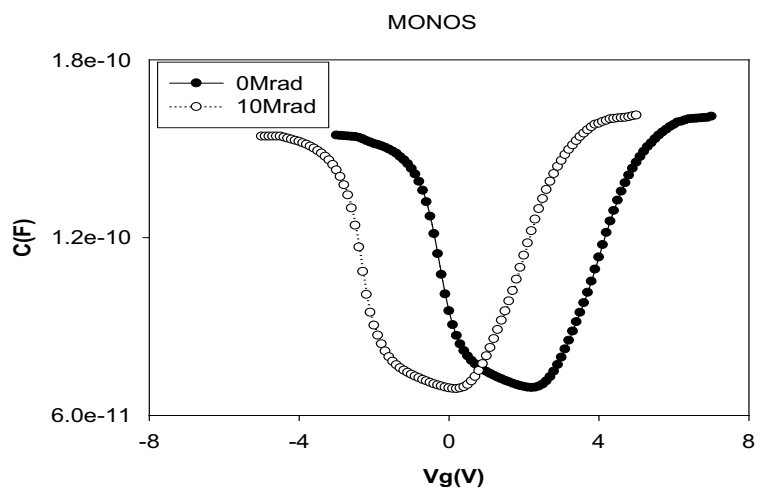

Figure 3. The C-V curve of MONOS device after $10 \mathrm{Mrad}$ Gamma ray irradiation.

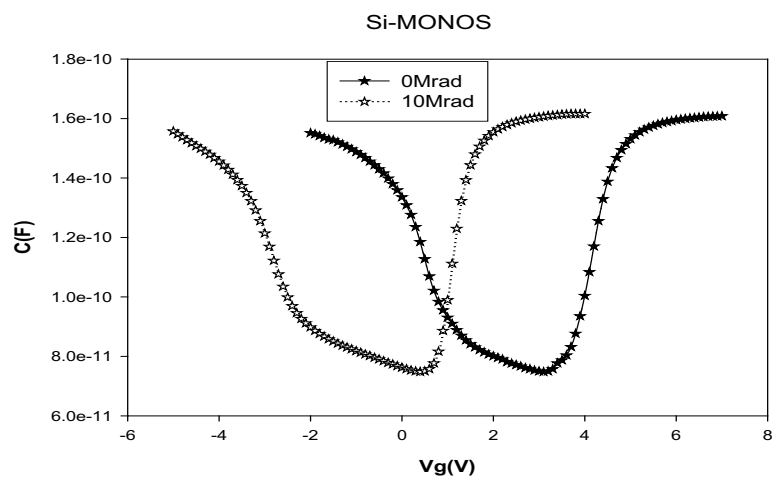

Figure 4. The C-V curve of Si-MONOS device after $10 \mathrm{Mrad}$ Gamma ray irradiation. 
MONOS IG-VG

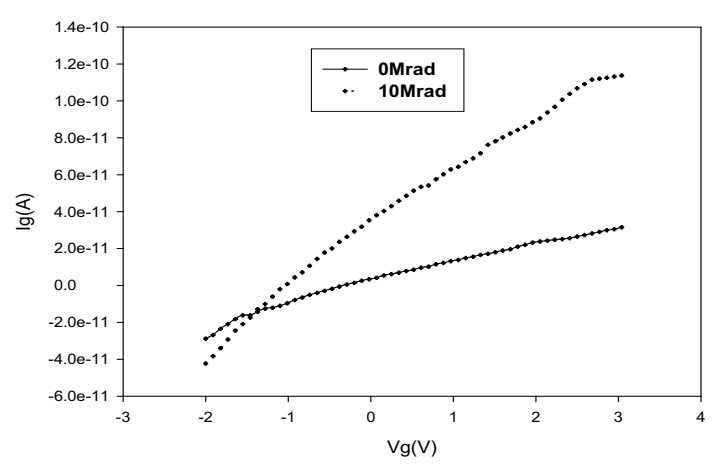

Figure 5. The gate current of MONOS device after 10Mrad gamma exposure.

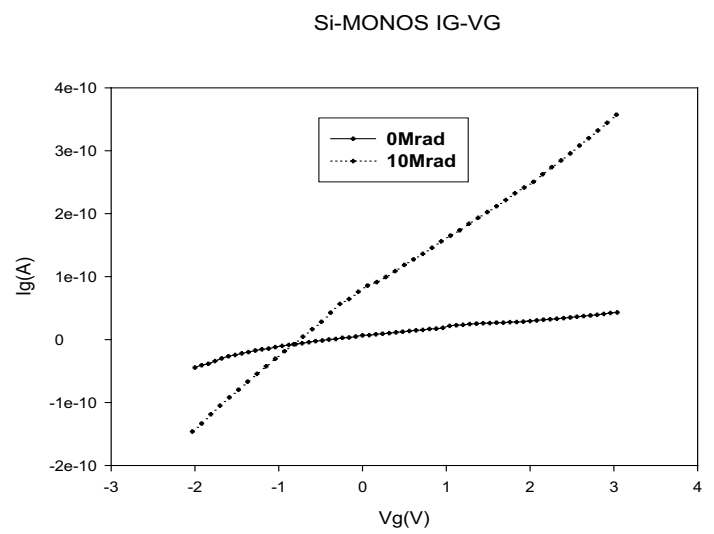

Figure 6. The gate current of Si-MONOS device after 10Mrad gamma ray exposure.

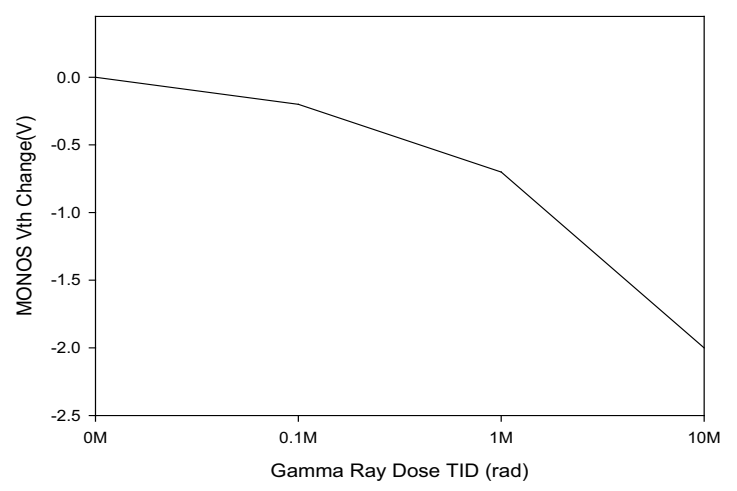

Figure 7. The dependencies of the $\mathrm{V}_{\mathrm{T}}$ decrease on gamma radiation TID for MONOS

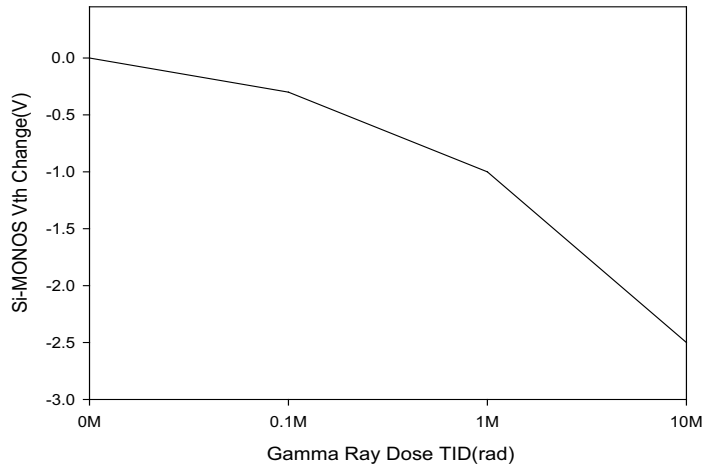

Figure 8. The dependencies of the $\mathrm{V}_{\mathrm{T}}$ decrease on gamma radiation TID for Si-MONOS

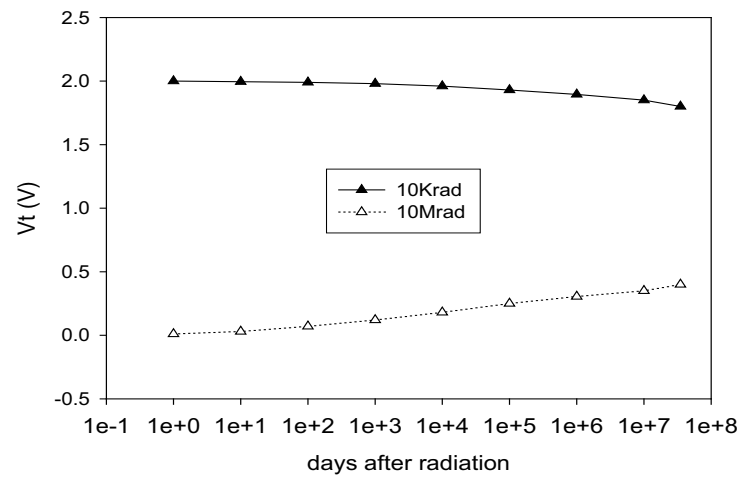

Figure 9. The $\mathrm{V}_{\mathrm{T}}$ decay vs time of MONOS after $10 \mathrm{Mrad}$ gamma irradiation

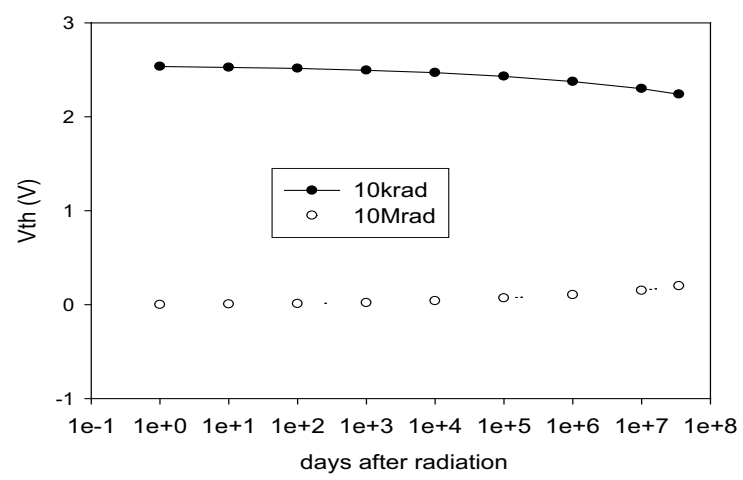

Figure 10. The $\mathrm{V}_{\mathrm{T}}$ decay vs time of Si-MONOS after 10Mrad gamma irradiation 